\title{
Pareto optimal allocation of fault current limiter based on immune algorithm considering cost and mitigation effect
}

\author{
Baichao CHEN ${ }^{1}$, Liangliang WEI ${ }^{1}$, Yuanzhe ZHU $^{1}$, Yongheng ZHONG ${ }^{1}$, \\ Jiaxin YUAN $^{1}$, Yang LEI ${ }^{1}$
}

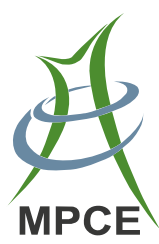

\begin{abstract}
This paper presents a multi-objective Pareto optimal method for allocation of fault current limiters based on an immune algorithm, which takes into account two objectives of the cost and fault current mitigation effect. A sensitivity factor calculation method based on the rate of fault current mitigation is proposed to reduce the search space and improve the efficiency of the algorithm. In this approach, the objective functions related to the cost and fault current mitigation effect are established. A modified inversion operator based on equal cost is proposed to converge to global optimal solutions more effectively. The proposed algorithm is tested on the IEEE 39-bus system, and obtains the Pareto optimal solutions, from which the user can select the most suitable solutions according to the preferences and relative importance of the
\end{abstract}

CrossCheck date: 05 August 2016

Received: 2 November 2015/Accepted: 5 August 2016/Published online: 9 November 2016

(C) The Author(s) 2016. This article is published with open access at Springerlink.com

$\triangle$ Liangliang WE

w1190623@163.com

Baichao CHEN

whgycbc@163.com

Yuanzhe ZHU

782824874@qq.com

Yongheng ZHONG

zyhwhuer@126.com

Jiaxin YUAN

yjx98571@163.com

Yang LEI

leyon91@163.com

1 School of Electrical Engineering, Wuhan University, Wuhan 430072, China objective functions. Simulation results are used to verify the proposed method.

Keywords FCL optimal allocation, Fault current mitigation effect, Modified inversion operator, Pareto optimal solutions, Immune algorithm

\section{Introduction}

With the increasing scale of power systems and continuous growth of distributed energy, fault currents have been larger and, in some case, beyond the rated ultimate capacity of existing circuit breakers (CBs). As a result, there is a serious threat to security and reliability of power systems. There are several traditional approaches to limiting the fault current, such as the air-core reactor, highimpedance transformer and network splitting. However, these approaches have some drawbacks [1]. The fault current limiter (FCL) has been one of the most promising methods to limit fault currents in power systems. There are several different types of FCL, including the solid-state, quenching superconducting and saturated-core fault current limiters [2-5]. The FCLs can be regarded as a variableimpedance device in series with the lines to provide low steady-state impedance under normal conditions and high limiting impedance during fault conditions [6]. But in power systems, the advantages of FCLs depend on the number, location and impedance of FCLs. Therefore, considering the efficiency and mitigation effect, the optimal allocation of FCLs, in terms of their number, locations and impedances, has become a difficult multi-objective problem. Some research has been published on optimal allocation of FCLs [7-18]. 
If the grid is small and has few branches, the enumeration method could be used to optimize the allocation of FCLs [7-9]. But if the grid is larger and has more buses exceeding fault current limits, the optimal location, number and impedance of FCLs would be difficult to find. Therefore, the enumeration method would be a very large computation, and not practicable.

As a result, various optimization algorithms have been proposed for the optimal allocation of FCLs [10-18]. A particle swarm optimization (PSO) method was employed to search for the optimal allocation of FCLs in [10], and a harmony search algorithm was used in [11]. References $[12,13]$ address the optimal allocation and sizing of FCLs in distribution systems. An optimal allocation method of superconducting fault current limiters (SFCLs) for reducing fault currents and mitigating voltage sag is proposed in [14]. An iterative mixed integer nonlinear optimization method was proposed for optimal allocation of FCL allocation in [15-17].

Most previous research transforms a multi-objective problem into a single-objective one by using weight coefficients. Since optimization results were largely affected by weight coefficients, and previous works took into consideration only the cost of FCLs, these results were not accurate.

In fact, the user should consider the cost of FCLs and their effect on fault current mitigation in the power grid. The cost is a function of total impedance and the number of FCLs. The fault current mitigation effect is a function of fault current reduction after installing FCLs. Moreover, the objective functions of the cost and fault current mitigation effect are conflicting. Thus, the user must select the most suitable solutions according to preferences and relative importance of the objective functions.

In [18], a sensitivity factor method was proposed to reduce search space. But the magnitude of the sensitivity factor used in [18] depends on the fault current at every bus. Comparing the factors of buses is not accurate. This sensitivity factor does not accurately assess the fault currents across all buses. Therefore, this paper proposes a new sensitivity factor calculation method based on the rate of fault current mitigation. It is not impacted by the absolute fault current at every bus and represents a fair assessment among buses.

This paper proposes a new method to optimize the multi-objective Pareto allocation of the FCLs by using the immune algorithm. Due to the sensitivity factor calculation, optimal FCL locations are easier to find, compared to impedances of FCLs. However, with the randomness of inversion and variation for conventional IA, the optimal installation locations of new FCLs would be not optimal after variation or inversion, resulting in converging to local minima. Therefore, a modified inversion operator based on equal cost is proposed to improve the convergence of the IA.

Compared to traditional methods, this paper considers two objectives of the cost and fault current mitigation effect. A new sensitivity factor calculation and a modified inversion operator are proposed to make the IA converge to the optimal solutions better. The results obtained by testing the IEEE 39-bus test system demonstrate the proposed method is effective. And the methods to improve the efficiency of calculation are discussed and analyzed in detail.

\section{Mathematical model and optimal allocation}

\subsection{Self-impedance correction}

In power systems, the three-phase fault is the most serious kind and is always used to determine the rated capacity of circuit breakers. The basic principle of short circuit calculation shows, for a three-phase fault at bus $k$, the fault current at bus $k$ can be calculated by

$I_{k}=\frac{E_{k}^{(0)}}{Z_{k k}}$

where $I_{k}$ is the three-phase fault current at bus $k ; Z_{k k}$ is the self-impedance of bus $k$ in the impedance matrix $\boldsymbol{Z}_{\text {bus }} ; E_{k}^{(0)}$ is the pre-fault voltage of bus $k$.

$E_{k}^{(0)}$ can be obtained from the power flow calculation under normal operations. The impedance matrix $\boldsymbol{Z}_{\text {bus }}$ is inverse of the admittance matrix $\boldsymbol{Y}_{\text {bus }}$, considering the transient reactance of generators.

From (1), the fault current $I_{k}$ is inversely proportional to the self-impedance $Z_{k k}$. If a FCL with limiting impedance $Z_{\mathrm{FCL}}$ is installed on a branch $i j$ between buses $i$ and $j$ (the branch impedance between buses $i$ and $j$ is $Z_{i j}$, when a three-phase fault happens, the FCL with limiting impedance $Z_{\mathrm{FCL}}$ is inserted in the branch $i j$, and the original impedance matrix $\boldsymbol{Z}_{\text {bus }}$ should be modified. Figure 1 shows the Thevenin equivalent circuit by paralleling the equivalent impedance $Z_{\mathrm{F}}$ in the branch $i j$.

The equivalent impedance $Z_{\mathrm{F}}$ can be calculated by

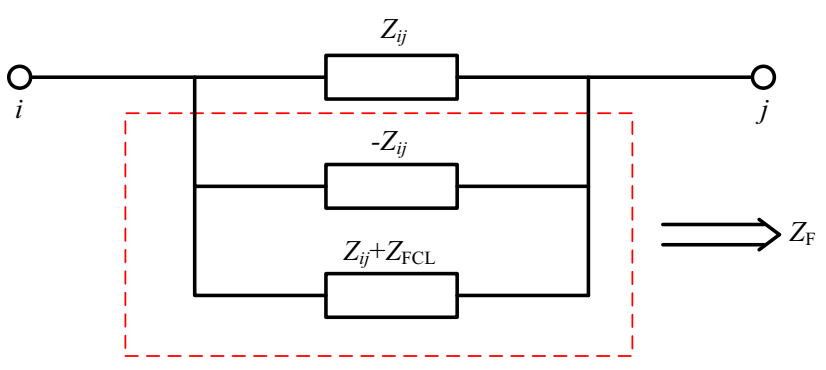

Fig. 1 Equivalent circuit when a FCL is inserted in branch $i j$ 
$Z_{\mathrm{F}}=\left(-Z_{i j}\right) / /\left(Z_{i j}+Z_{\mathrm{FCL}}\right)=-Z_{i j} \frac{Z_{i j}+Z_{\mathrm{FCL}}}{Z_{\mathrm{FCL}}}$

Therefore, when the FCL with impedance $Z_{\mathrm{FCL}}$ is inserted in the branch $i j$, the modified self-impedance $Z_{k k}^{\prime}$ of bus $k$ is

$Z_{k k}^{\prime}=Z_{k k}-\frac{\left(Z_{k i}-Z_{k j}\right)^{2}}{Z_{i i}+Z_{j j}-2 Z_{i j}+Z_{\mathrm{F}}}=Z_{k k}+\Delta Z_{k k}$

\subsection{Sensitivity factor calculation based on the rate of fault current mitigation}

As discussed in Section 1, if a power system is sophisticated and has many buses exceeding fault current limits, the enumeration method is very time-consuming and inefficient. As a result, a sensitivity factor calculation is used to screen the better candidate locations of FCLs [18], and this paper presents a new sensitivity factor calculation method based on the rate of the fault current mitigation.

From (2), when a FCL with impedance $Z_{\mathrm{FCL}}$ is installed in a branch $i j$ between the buses $i$ and $j$, for a three-phase fault at bus $k$, the rate of fault current mitigation at bus $k$ can be calculated by

$\phi_{i j}^{k}=\frac{I_{k}^{\prime}-I_{k}}{I_{k}}=\frac{\frac{V_{k}^{(0)}}{Z_{k k}+\Delta Z_{k k}}-\frac{V_{k}^{(0)}}{Z_{k k}}}{\frac{V_{k}^{(0)}}{Z_{k k}}}=-\frac{\Delta Z_{k k}}{Z_{k k}+\Delta Z_{k k}}$

where $I_{k}$ is the original fault current at bus $k ; I_{k}^{\prime}$ is the fault current at bus $k$ after installing the FCL in the branch $i j$; $\Delta Z_{k k}$ is the change of self-impedance of bus $k$ due to the FCL with impedance $Z_{\mathrm{FCL}}$ as shown in (3).

From (4), the rate of fault current mitigation at every bus can be calculated for every FCL placement. If the impedance $Z_{\mathrm{FCL}}$ is identical for all FCLs, the rate of fault current mitigation is referred to as the sensitivity factor. The factor is relative and not impacted by the fault current of every bus, and therefore represents a fair assessment among buses. If the value of rate is larger, it means that installing the FCL in this branch is more effective for mitigating fault currents. Therefore, the branches with the largest $a$ rates can be screened to be the best candidate locations, where $a$ is the number of candidate locations of a bus.

\section{Multi-objective function for optimal allocation}

\subsection{Objective function for cost of FCLs}

The first objective function for optimal allocation of FCLs is minimizing the cost of FCLs. As FCLs with larger impedance have a higher cost of materials and installation, the cost of FCLs is represented by the sum of total impedance of FCLs and their installation cost. The installation cost is proportional to the number of FCLs. Therefore, the cost of FCLs can be expressed as follows:

$M=\sum_{i=1}^{N_{\mathrm{FCL}}} Z_{\mathrm{FCL}}(i)+\omega N_{\mathrm{FCL}}+F_{\mathrm{a}}$

$Z_{\min } \leq Z_{\mathrm{FCL}}(i) \leq Z_{\max }$

$I_{k}^{\prime}<I_{\mathrm{G}}$

where (6) are the impedance constraints of FCLs and (7) are fault current constraints; $M$ represents the cost of FCLs on condition that target fault currents are achieved; $Z_{\mathrm{FCL}}(i)$ is the impedance of FCL $i ; N_{\mathrm{FCL}}$ is the number of FCLs; $\omega$ is the coefficient of the installation $\operatorname{cost} ; F_{\mathrm{a}}$ is the penalty factor of fault current constraints, where $F_{\mathrm{a}}=$ constant $b$ if $I_{k}^{\prime}>I_{\mathrm{G}}$ else $0 ; I_{\mathrm{G}}$ is the target value of fault current, so that the penalty factor $F_{\mathrm{a}}$ enforces fault current constraints of all buses.Therefore, the first objective function for optimal allocation of FCLs can be formulated as

$F_{1}\left(Z_{\mathrm{FCL}}(i), N_{\mathrm{FCL}}\right)=\frac{1}{\sum_{i=1}^{N_{\mathrm{FCL}}} Z_{\mathrm{FCL}}(i)+\omega N_{\mathrm{FCL}}+F_{\mathrm{a}}}$

which is simply the inverse of the cost of FCLs $M$.

\subsection{Objective function for fault current mitigation}

In the most previous studies, the objective for optimal allocation of FCLs is just making the fault currents of all buses below the target current $I_{\mathrm{G}}$ or the rated ultimate currents of circuit breakers (CBs). This paper presents a method to consider the total fault current mitigation effect after installing FCLs, making the fault currents of all buses approach the target current $I_{\mathrm{G}}$ within certain margins. The maximum margin value of fault currents is defined as $I_{\mathrm{M}}$. This paper assumes it is equal to $90 \%$ of the target value $I_{\mathrm{G}}$. The second objective function for optimal allocation of FCLs is making the buses exceeding fault current limits, or with insufficient fault current margin, approach the maximum margin $I_{\mathrm{M}}$ as closely as possible. If the fault currents of these buses are farther below the target current $I_{\mathrm{G}}$, the total fault current mitigation effect after installing the FCLs is better.

The fault mitigation current effect of bus $k$ can be expressed as

$\eta_{k}=\frac{I_{\mathrm{G}}-I_{k}^{\prime}}{I_{\mathrm{G}}}=\frac{\frac{U_{k}^{(0)}}{Z_{\mathrm{G} k}}-\frac{U_{k}^{(0)}}{Z_{k k}}}{\frac{U_{k}^{(0)}}{Z_{\mathrm{G} k}}}=\frac{Z_{k k}^{\prime}-Z_{\mathrm{G} k}}{Z_{k k}^{\prime}}$

where $Z_{\mathrm{G} k}$ is the target self-impedance of bus $k$ corresponding to the target current, $Z_{\mathrm{G} k}=V_{k}^{(0)} / I_{\mathrm{G}} ; Z_{k k}^{\prime}$ is the self-impedance of bus $k$ after installing FCLs, 
$Z_{k k}^{\prime}=V_{k}^{(0)} / I_{k}^{\prime} ; Z_{\mathrm{M} k}$ is the margin value of the self-impedance of bus $k$ corresponding to the maximum margin value of the fault current $I_{\mathrm{M}}$, given by $Z_{\mathrm{M} k}=V_{k}^{(0)} / I_{\mathrm{M}}$. When $Z_{k k}^{\prime} \geq Z_{\mathrm{M} k}$, the algorithm enforces $Z_{k k}^{\prime}=Z_{\mathrm{M} k}$, which means the fault current at bus $k$ has been met the margin requirement. Constraining the impedance below $Z_{\mathrm{M} k}$ can avoid the impedance of FCLs increasing and can thereby reduce the cost.

Therefore, the second objective function for optimal allocation of FCLs, representing the fault current mitigation effect, can be formulated as

$F_{2}\left(Z_{\mathrm{FCL}}(i), N_{\mathrm{FCL}}\right)=\sum_{k=1}^{N_{k}} \varepsilon_{k} \eta_{k}$

$\varepsilon_{k}$ is the weight coefficient of mitigation effect. If the fault current at bus $k$ exceeds fault current limits, $\varepsilon_{k}=1$. If the fault current at bus $k$ has insufficient fault current margin, $\varepsilon_{k}=I_{k} / I_{\mathrm{G}}$. Thus, if the values of $\varepsilon_{k}$ are equally balanced between buses exceeding fault current limits and buses with insufficient fault current margin, the total mitigation effect of the bus exceeding fault current limits is better.

\subsection{Multi-objective function of optimal allocation and Pareto-optimal solutions}

This paper proposes a multi-objective optimal method for the allocation of FCLs, considering the cost of FCLs and their fault current mitigation effect on the overall power grid. The multi-objective function can be expressed as

$F=\left\{\begin{array}{l}\max \left(F_{1}\right) \\ \max \left(F_{2}\right) \\ Z_{\min } \leq Z_{\mathrm{FCL}}(i) \leq Z_{\max } \\ I_{k}<I_{\mathrm{G}}\end{array}\right.$

The optimal allocation of the FCLs is based on conflicting objectives of minimizing the cost of FCLs and maximizing their fault current mitigation effect. As discussed in Section 1, methods for transforming the multi-objective problem into a single objective problem are not accurate because they are greatly influenced by the weight coefficient balancing these objectives. This paper presents a method based on Pareto-optimal solution to solve the multi-objective problem.

The Pareto optimal solution is derived from the multiobjective problem defined in (12) [19, 20]. Defining $X$ as the problem solution space, then only if there is no other solution $v$ in the $X$ for which $\boldsymbol{F}(v) \geq \boldsymbol{F}(x)$, the Pareto optimal solution of the multi-objective problem is $x \in X$.

$$
\left\{\begin{array}{cl}
\max & \boldsymbol{F}(x)=\left[f_{1}(x), f_{2}(x), \ldots, f_{n}(x)\right]^{\mathrm{T}} \\
\text { s.t. } & \boldsymbol{h}(x)=\mathbf{0} \\
& \boldsymbol{g}(x) \leq \mathbf{0}
\end{array}\right.
$$

where $\boldsymbol{F}(x)$ is the objective function vector.; $\boldsymbol{h}(x)$ and $\boldsymbol{g}(x)$ are equality and inequality constraints.

The Pareto optimal solution is not the only, but a Pareto optimal solution set. Therefore, the user can select the most suitable solutions according to the preferences and relative importance of objective functions.

\section{Procedure based on immune algorithm}

The immune algorithm is a kind of intelligent optimization algorithm based on features of biological immune systems [20-22]. It considers a problem as invading antigen. The optimal solution of the problem is regarded as the antibody produced by the immune system. The immune algorithm has the advantages of fast search, stronger local and global search ability, self-regulation, and quick converged ability. Thus, this paper proposes the immune algorithm for the multi-objective optimal allocation of FCLs. Figure 2 is the procedural flowchart of optimal allocation of FCLs based on immune algorithm.

\subsection{Selecting candidate locations}

Target values of the fault current and maximum margin value should be provided. Three-phase fault current calculation should be done for every bus of the test system to find the buses exceeding fault current limits and with insufficient fault current margin. Candidate locations should be selected based on the sensitivity factor calculation.

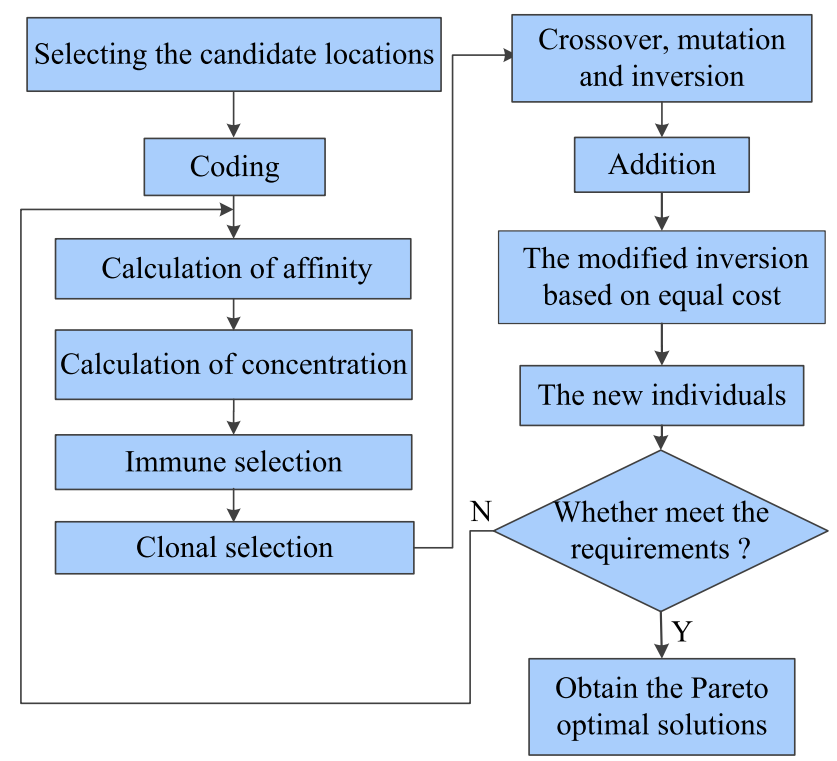

Fig. 2 Flow chart of the proposed optimum algorithm 


\subsection{Coding of FCL impedances}

The optimal allocation of FCLs should consider the installation locations, number and impedance. Therefore, this paper proposes a simple coding of these parameters, in which genes of a chromosome represent the impedances of FCLs. The position of each gene indicates the installation location of the FCL. If the impedance $Z=0$ for location $k$ then there is no FCL installed in location $k$. The coding can be expressed as

$C_{m}=\left(Z_{m 1}, Z_{m 2}, Z_{m 3}, \ldots, Z_{m n} \ldots,\right)$

where $C_{m}$ represents the chromosome of individual $m ; Z_{m n}$ is the impedance of FCL on the gene $n$ of individual $m$.

\subsection{Conventional operations of immune algorithm}

The conventional operations of immune algorithm include crossover, mutation, inversion, and addition, the calculation of affinity, immune selection and clonal selection [22].

\subsection{Modified inversion based on equal cost}

Due to the sensitivity factor calculation, the optimal installation locations are more easily searched than the impedance of FCLs. However, with the randomness of inversion and variation for the conventional Immune Algorithm, the optimal installation locations of new individuals would be not optimal after these operations, resulting in the algorithm being trapped by local minima. Therefore, this paper proposes the modified inversion operator based on equal cost to make the algorithm converge to the optimal solution better. The modified inversion operator based on equal cost has the property that, when not changing the optimal location of FCLs, the procedure keeps the cost or the total impedance of FCLs the same, and randomly selects two locations from the non-zero locations to form the inverse. The modified inversion operator based on equal cost can be shown as

$$
\begin{aligned}
& \left\{\begin{aligned}
C_{1}= & \left(0, \ldots, Z_{\mathrm{a}}, \ldots, Z_{\mathrm{b}}, \ldots, 0\right) \\
& \downarrow \text { Inversion } \\
C_{2}= & \left(0, \ldots, Z_{\mathrm{c}}, \ldots, Z_{\mathrm{d}}, \ldots, 0\right)
\end{aligned}\right. \\
& \left\{\begin{array}{l}
Z_{\mathrm{c}}=k Z_{\mathrm{a}}+(1-k) Z_{\mathrm{b}} \\
Z_{\mathrm{d}}=(1-k) Z_{\mathrm{a}}+k Z_{\mathrm{b}}
\end{array}\right.
\end{aligned}
$$

where $k$ is an arbitrary decimal between 0 and 1 .

\section{Case validation and discussions}

The IEEE 39-bus test system is chosen to validate the optimal allocation method of the FCLs proposed by this paper, as shown in Fig. 3. This paper assumes the target fault current $I_{\mathrm{G}}=70.0$ p.u., the maximum margin current is $I_{\mathrm{M}}=0.9 I_{\mathrm{G}}=63.0$ p.u., the coefficient of the installation cost $\omega=5$, and the impedance constraint of the FCLs is $0 \leq Z_{\mathrm{FCL}} \leq 0.1$ p.u.

With the three-phase fault current calculation, the test system has three buses exceeding fault current limits and two buses with insufficient fault current margin: buses 2, 3, 16, 17 and 39 (the red buses in Fig. 3). The corresponding fault currents of the buses are shown in Table 1. Due to the generator transient reactance of bus 39 being too small, the fault current at bus 39 is very large. Considering the most economic solution, interruption could be used to limit the fault current at bus 39 . Thus, this paper only mitigates the fault currents for buses 2, 3, 16 and 17 by installing the FCLs.

\subsection{Comparison of fault current calculation between algorithm and PSASP}

Figure 4 shows the results of fault current calculation for the theoretical algorithm and Power System Analysis Software Package (PSASP). It can be seen that, the error between the theoretical calculation and PSASP is very small when there is no FCL. When a FCL with the impedance of 0.02 p.u. is installed in branch 1-2, the result of theoretical calculation is also almost equal to that of PSASP, and the fault currents at bus 2, bus 3 and bus 39 are limited. Hence, the results verify the correctness of the theoretical calculation.

\subsection{Selecting candidate locations}

Assuming $Z_{\mathrm{FCL}}=0.01$ p.u., the sensitivity factor is calculated at every bus, and candidate locations are

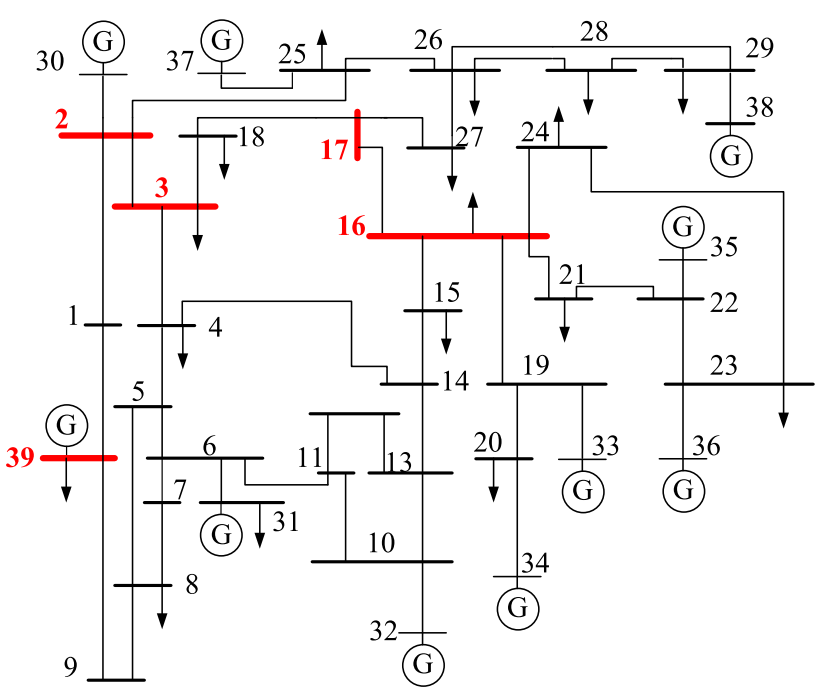

Fig. 3 IEEE 39-bus test system 
Table 1 Fault levels of system

\begin{tabular}{ll}
\hline Bus number & Fault level \\
\hline 2 & 75.87 \\
3 & 63.19 \\
16 & 71.83 \\
17 & 63.06 \\
39 & 191.81
\end{tabular}

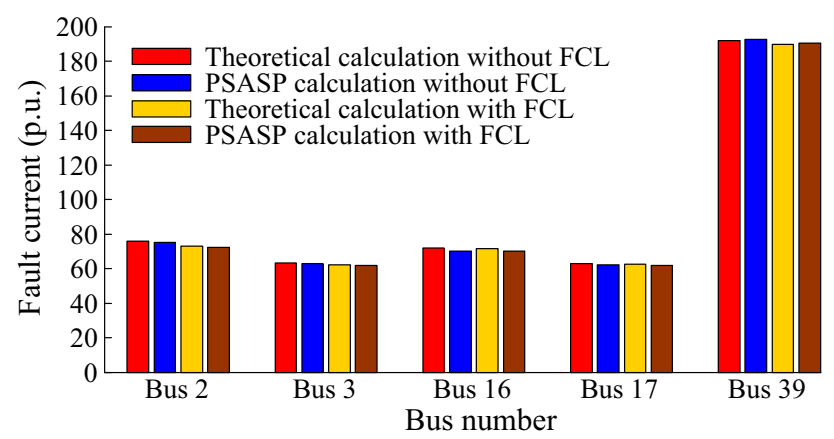

Fig. 4 Results of fault current calculation for the theoretical algorithm and PSASP

selected based on the sensitivity factor calculation. Setting the number of candidate locations per bus to $a=8$ the candidate locations of FCLs are shown in Table 2.

By removing the repetitive locations, the number of total candidate locations is 17 in this example.

\subsection{Results of optimal allocation}

This paper uses the immune algorithm for the optimal allocation of the FCLs. The parameters of the algorithm are set out in Table 3.

Two cases with a fixed number and with an unfixed number of FCLs are considered to obtain the Pareto optimal solutions. The Pareto optimal solutions in the form of Pareto frontiers are shown in Fig. 5. The horizontal axis shows the objective function of the cost, and the vertical axis shows the objective function of the mitigation effect. The blue, green and purple curves respectively represent
Table 3 Parameters of immune algorithm

\begin{tabular}{ll}
\hline Parameters & Value \\
\hline Number of population & 400 \\
Maximum iteration number & 50000 \\
Length of chromosome & 17 \\
Penalty factor $F_{\mathrm{a}}$ & 10000 \\
Rate of crossover $P_{\mathrm{c}}$ & 0.55 \\
Rate of mutation $P_{\mathrm{m}}$ & 0.006 \\
Rate of inversion $P_{\text {con }}$ & 0.005 \\
Rate of modified inversion $P_{\text {icon }}$ & 0.05 \\
\hline
\end{tabular}

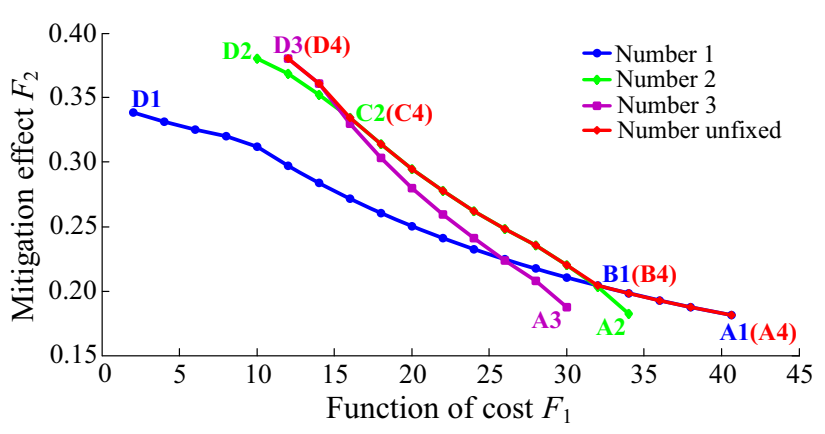

Fig. 5 Pareto optimal solutions of optimal allocation of FCLs

the Pareto optimal solutions when there are 1,2 and 3 FCLs respectively. The red curve is the Pareto optimal solution with an unfixed number of FCLs.

1) Relationship of the cost and the mitigation effect

It can be seen from Fig. 5, the objective functions of the cost and mitigation effect are approximately in inverse proportion, bearing in mind that the cost of FCLs $M$ is the reciprocal of the objective function of the cost. If the cost is less, the mitigation effect is worse. The better the mitigation effect required, the greater the cost.

2) Analysis of the Pareto optimal solutions

The point A1 (A4) in Fig. 5 is the maximum value of objective function of the cost without considering the mitigation effect and just meeting the fault current constraints. Thus, point A1 (A4) is the most conservative case of optimal allocation, ensuring the fault currents at all

Table 2 Candidate locations of FCLs

\begin{tabular}{ll}
\hline Bus number & Candidate locations (rate of fault current mitigation \%) \\
\hline 2 & $2-3(7.86) ; 30-2(7.59) ; 2-25(6.41) ; 39-1(4.07) ; 1-2(3.87) ; 16-17(3.14) ; 3-4(2.7) ; 7-25(2.24)$ \\
3 & $2-3(14.45) ; 3-4(7.18) ; 17-18(7.08) ; 3-18(6.98) ; 16-17(5.02) ; 30-2(3.23) ; 4-5(2.59) ; 16-19(1.59)$ \\
16 & $16-17(8.54) ; 16-19(5.97) ; 14-15(4.05) ; 15-16(3.9) ; 3-18(3.27) ; 17-18(3.21) ; 21-22(2.74) ; 2-3(2.65)$ \\
17 & $16-17(19.73) ; 3-18(7.43) ; 17-18(7.32) ; 2-3(3.54) ; 16-19(3.52) ; 26-27(3.33) ; 27-17(3.21) ; 30-2(1.63)$ \\
\hline
\end{tabular}


buses only just meet the fault current constraints: $I_{k}^{\prime}<I_{\mathrm{G}}$. The solutions of this case are as follows: a FCL with an impedance of 0.0196 p.u. is installed in branch 2-3. The mitigation effect is: the fault current at buses 2, 3, 16 and 17 respectively is limited to 69.99 p.u., 69.96 p.u., 63.0 p.u. and 63.0 p.u.

It can also be seen from Fig.5 that, if the objective function of the cost is subject to $32.0 \leq F_{1} \leq 40.65$ (B4A4), installing one FCL is optimal. If $16.0 \leq F_{1} \leq 32.0$ (C4-B4), installing two FCLs is optimal. And installing three FCLs is optimal when $F_{1}<16.0$. These results show how the installation cost of the FCLs and the required mitigation effect are balanced. Moreover, the points D4, D2 and D3 are the maximum values of the mitigation effect. At these points, the fault currents at all buses can be limited to 63.0 p.u.. And the cost of installing three FCLs (D4 or D3) is minimal to achieve the best mitigation effect. The optimal solutions of this case is as follows: the first FCL with impedance of 0.0181 p.u. is installed in branch 2-3, the second FCL with impedance of 0.0235 p.u. is installed in branch 16-17, the third FCL with impedance of 0.0221 p.u. is installed in branch $30-2$. When only one FCL is used (D1), the impedance of the FCL would be very large, which is neither within the impedance constraints, nor economic.

3) Pareto optimal solutions between different fault current goals

The Pareto optimal solutions between different target fault currents are shown in Fig. 6. The blue curve shows the optimal solutions when $I_{\mathrm{G}}=70.0$ p.u., while the red curve presents the optimal solutions when $I_{\mathrm{G}}=63.0$ p.u. The smaller is the value of the target fault current, the larger is the cost of FCLs to achieve it, and the better is the corresponding mitigation effect.

Therefore, after the Pareto optimal solutions have been obtained by the immune algorithm, the user can select the most suitable solutions according to preferences and importance of the objective functions.

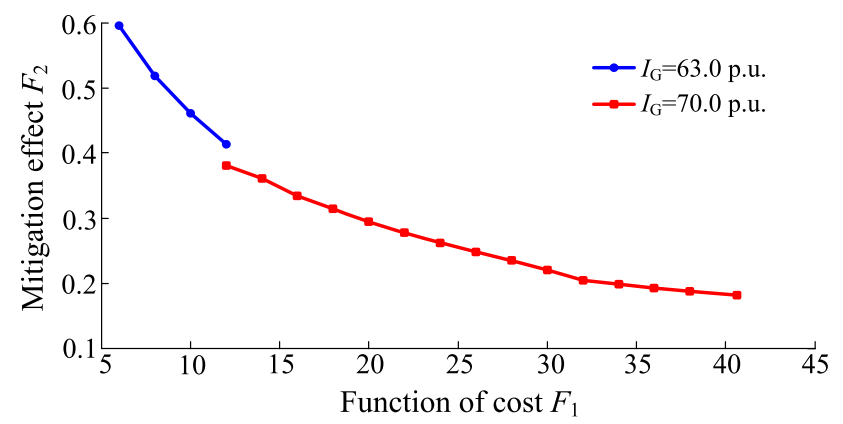

Fig. 6 Comparison of Pareto optimal solutions between different fault current goals

\section{Discussion for improving efficiency of calculation}

\subsection{Comparison between sensitivity factor method and traditional whole network search}

Figure 7 shows the performance comparison between sensitivity factor method and traditional whole network search when $F_{1}=20.0$ and $I_{\mathrm{G}}=70.0$ p.u. It can be seen that two methods can both converge to the optimal solution, but compared with the traditional whole network search, the sensitivity factor method proposed in this paper can reduce the search space, amount of calculation and number of iterations and, therefore, it can improve the efficiency of calculation.

\subsection{Analysis of modified inversion based on equal cost}

As convergence of the conventional immune algorithm could easily fall into local minima, the modified inversion based on equal cost proposed by this paper can make the algorithm converge reliably to the optimal solutions. Table 4 and Fig. 8 compare the conventional immune algorithm and the algorithm with modified inversion based on equal cost. This paper analyzes two optimal results when $F_{1}=20.0, I_{\mathrm{G}}=70.0$ p.u. and $F_{1}=14.0, I_{\mathrm{G}}=70.0$ p.u.

It can be seen from Table 4 and Fig. 8, the total impedance of FCLs resulting from the modified and conventional algorithms is the same. In other words, the cost reached by two algorithms is equal. But the modified algorithm gives a better mitigation effect than the conversional algorithm. This suggests that the modified algorithm proposed by this paper can converge to optimal solutions more reliably, avoiding local minima.

According to many tests, the rate of modified inversion $P_{i \text { con }}$ in the range of 0.05 to 0.1 is appropriate. If the rate is too small, the impact of the modified inversion is not effective. And the larger rate is not necessary.

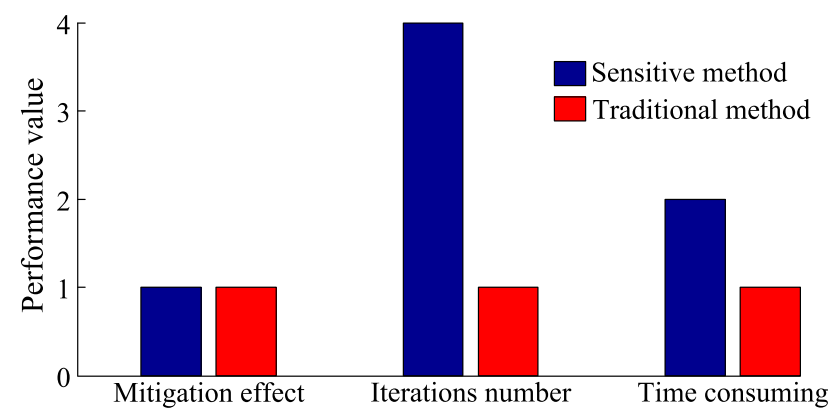

Fig. 7 Performance comparison of two methods 
Table 4 Comparison of conventional and modified immune algorithm

\begin{tabular}{|c|c|c|c|c|}
\hline Goals & Methods & Impedances of FCLs & Mitigation effect & Convergence \\
\hline \multirow{4}{*}{$\begin{array}{l}F_{1}=20.0 \\
I_{\mathrm{G}}=70 \text { p.u. }\end{array}$} & \multirow[t]{2}{*}{ Conventional } & $0.0196(2-3)$ & \multirow[t]{2}{*}{0.2947} & \multirow[t]{2}{*}{ Local optimum } \\
\hline & & $0.0203(16-17)$ & & \\
\hline & \multirow[t]{2}{*}{ Modified } & $0.0188(2-3)$ & \multirow[t]{2}{*}{0.2948} & \multirow[t]{2}{*}{ Global optimum } \\
\hline & & $0.021(16-17)$ & & \\
\hline$F_{1}=14.0$ & \multirow[t]{3}{*}{ Conventional } & $0.0224(2-3)$ & \multirow[t]{3}{*}{0.3602} & \multirow[t]{3}{*}{ Local optimum } \\
\hline \multirow[t]{5}{*}{$I_{\mathrm{G}}=70$ p.u. } & & $0.0223(16-17)$ & & \\
\hline & & $0.0117(2-30)$ & & \\
\hline & \multirow[t]{3}{*}{ Modified } & $0.0197(2-3)$ & \multirow[t]{3}{*}{0.3608} & \multirow[t]{3}{*}{ Global optimum } \\
\hline & & $0.0236(16-17)$ & & \\
\hline & & $0.0131(2-30)$ & & \\
\hline
\end{tabular}

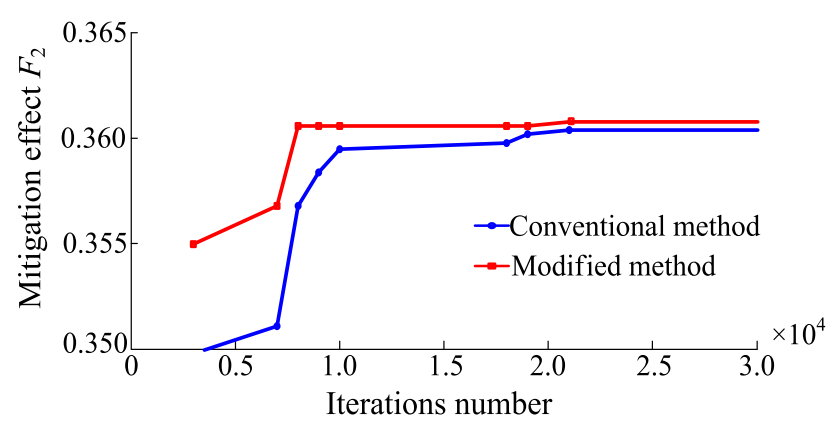

Fig. 8 Comparison of convergence between the conventional and modified immune algorithm

\subsection{Analysis of number of candidate locations of one bus}

According to the Pareto optimal solutions described above, the locations of FCLs are branch 2-3, branch 2-30, branch 16-17 and branch $16-19$ when $I_{\mathrm{G}}=70.0$ p.u. or $I_{\mathrm{G}}=63.0$ p.u., and the rates of fault current mitigation of these four branches are the top two in bus 2 and bus 16 $(a \leq 2)$. The total number of candidate locations would influence the optimal results and efficiency of calculation. When the number of candidate locations is large, the optimal solution is effective, but finding it requires a large amount of calculation. When the number of candidate locations is too small, the optimal solution may be of poor quality. Therefore it is necessary to analyze the required number of candidate locations on a bus. Figure 9 shows the total number of candidate locations for different values of $a$. The number of high rates of fault current mitigation selected per bus for screening in the sensitivity factor calculation, as discussed in Section 2.2.

From Fig. 9, the candidate locations are branch 2-3 and branch 16-17 when $a=1$, not including all the optimal solutions. This paper analyzes the convergence and the efficiency of calculation when $a=2-8$. Figure 10 shows

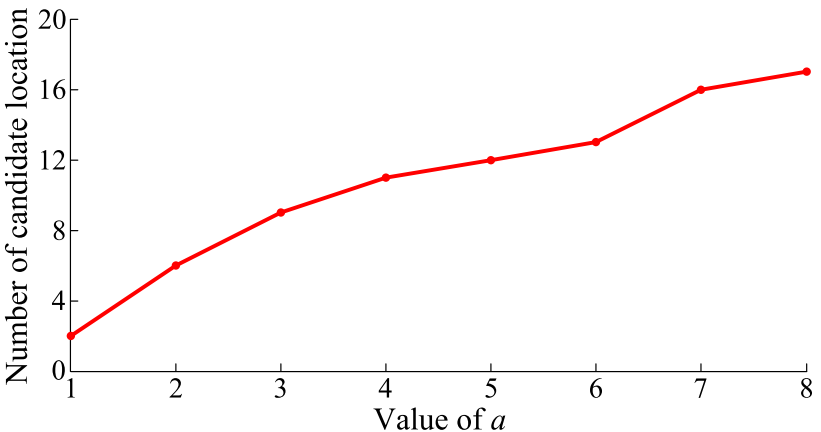

Fig. 9 Total number of candidate locations for different values of $a$ (the number of candidate locations per bus)

the maximum number of iterations to convergence for different values of $a$.

It can be seen from Fig. 10 that the maximum number of iterations will increase as the value of $a$ increases. Above $a=3$ or $a=4$, however, the optimal solutions do not improve significantly in their quality. Since the number of FCLs would increase when the target fault current decreases, the optimal solutions may not in the candidate locations and be of poor quality when $a=2$. Thus, $a=3$ or $a=4$ would be suitable values for the number of candidate location per bus.

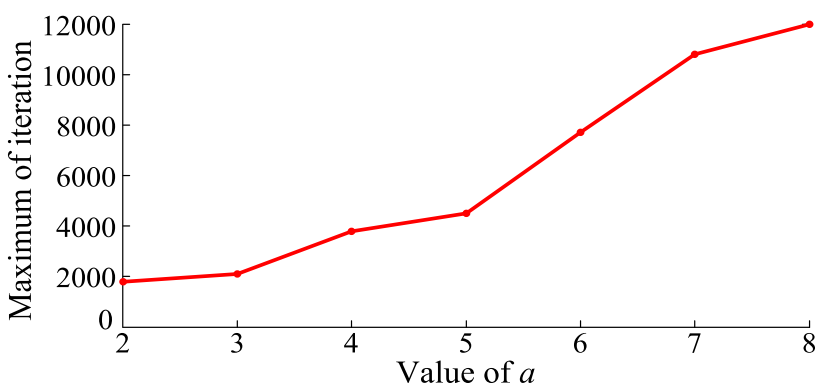

Fig. 10 Maximum number of iterations for different values of $a$ (the number of candidate locations per bus) 
Table 5 Optimal locations of FCL

\begin{tabular}{|c|c|c|c|}
\hline \multicolumn{2}{|l|}{$I_{\mathrm{G}}=70.0$ p.u. } & \multicolumn{2}{|l|}{$I_{\mathrm{G}}=63.0$ p.u. } \\
\hline Function of cost $F_{1}$ & Optimal locations & Function of cost $F_{1}$ & Optimal locations \\
\hline $32.0-40.0$ & Bus 2-3 & $8.0-12.0$ & Bus 2-3; Bus 16-17; Bus 2-30 \\
\hline $16.0-32.0$ & Bus 2-3; Bus 16-17 & Below 8.0 & Bus 2-3; Bus 16-17; Bus 2-30; Bus 16-19 \\
\hline Below 16.0 & Bus 2-3; Bus 16-17; Bus 2-30 & & \\
\hline
\end{tabular}

\subsection{Analysis of optimal allocation of FCLs}

From the analysis above, all optimal locations of FCLs are on branches with the highest rates of fault current mitigation. Table 5 shows the optimal locations of FCLs when $I_{\mathrm{G}}=70.0$ p.u. and $I_{\mathrm{G}}=63.0$ p.u.

It can be seen from Table 5, bus 2 and bus 16 are the buses exceeding fault current limits, and the fault current on bus 2 is larger than the fault current on bus 16. Branch 2-3 and branch 16-17 are respectively the branches with the highest rate of fault current mitigation on bus 2 and bus 16 . Increasing from $a=1$ to $a=2$, branch 2-30 and branch 16-19 have the next highest rates of fault current mitigation on bus 2 and bus 16, respectively. As a result, the following general rules can be proposed:

1) If the number of FCLs is one, the optimal location of FCLs is the location of the highest rate of fault current mitigation on the bus with the largest exceedance of fault current.

2) If the number of FCLs is two, the optimal locations of FCLs are the locations of the highest rate of fault current mitigation on the buses with the two largest exceedances of fault current.

3 ) If the number of FCLs is three, the optimal location of the third FCL is the location of the second largest rate of fault current mitigation on the bus with the largest exceedance of fault current.

4) If the number of FCLs is more, the optimal locations of FCLs might be determined by generalising these rules. Such rules, as proposed by this paper, would be significant in guiding the optimal allocation of FCLs.

\section{Conclusion}

The fault current limiter (FCL) is an effective and promising measure to limit fault currents and improve reliability of power systems. The optimal allocation of FCLs, which determines the optimal locations, number and impedance of FCLs, has become a difficult problem. This paper proposed a Pareto multi-objective optimization method based on Immune Algorithm to optimize the allocation of FCLs. Compared to previous optimization methods, this paper considered two objectives of the cost of FCLs and fault current mitigation effect. Moreover, a sensitivity factor calculation based on the rate of the fault current mitigation, and a modified inversion operator based on equal cost, were proposed to improve the calculation efficiency of the algorithm. They reduce the search space and amount of calculation, and improve the convergence to optimal solutions. The optimal algorithm proposed by this paper was verified by numerical simulation of the IEEE 39-bus test system, obtaining Pareto optimal solutions for the allocation of FCLs to achieve different fault current limits. The results demonstrate that the new optimization method have an importance role in power grid planning and the allocation of FCLs.

Acknowledgments This work was supported by National Natural Science Foundation of China (No. 50807041).

Open Access This article is distributed under the terms of the Creative Commons Attribution 4.0 International License (http:// creativecommons.org/licenses/by/4.0/), which permits unrestricted use, distribution, and reproduction in any medium, provided you give appropriate credit to the original author(s) and the source, provide a link to the Creative Commons license, and indicate if changes were made.

\section{References}

[1] Abramovitz A, Smedley KM, De LRF et al (2013) Prototyping and testing of a $15 \mathrm{kV} / 1.2 \mathrm{kA}$ saturable core hts fault current limiter. IEEE Trans Power Deliv 28(3):1271-1279

[2] Ghanbari T, Farjah E, Tashakor N (2016) Thyristor based bridge-type fault current limiter for fault current limiting capability enhancement. IET Gener Transm Distrib 10(9):2202-2215

[3] Pei X, Zeng X, Smith AC (2015) Resistive superconducting fault current limiter coil design using multistrand $\mathrm{MgB} 2$ wire. IEEE Trans Appl Supercond 25(3):1-5

[4] Lee H, Kang H (2016) Conceptual design of a resistive 154-kV/ 2-kA superconducting fault current limiter. IEEE Trans Appl Supercond 26(4):1-5

[5] Yuan J, Lei Y, Wei L et al (2015) A novel bridge-type hybrid saturated core fault current limiter based on permanent magnets. IEEE Trans Magn. doi:10.1109/INTMAG.2015.7157047

[6] Commins PA, Moscrop JW (2013) Analytical nonlinear reluctance model of a single-phase saturated core fault current limiter. IEEE Trans Power Deliv 28(1):450-457

[7] Didier G, Leveque J, Rezzoug A (2013) A novel approach to determine the optimal location of SFCL in electric power grid to 
improve power system stability. IEEE Trans Power Syst 28(2):978-984

[8] Mahender K, Subhash B et al (2013) Optimal positioning of superconducting fault current limiter in smart grid applications. In: Proceedings of the 2013 IEEE Asia Pacific conference on postgraduate research in microelectronics and electronics (PrimeAsia). Visakhapatnam, India, 19-21 Dec 2013, pp 257-270.

[9] Noman UDS, Islam B, Ahmed S (2015) Optimal positioning of bridge type superconducting fault current limiter in solar integrated electric power grid. In: Proceedings of IEEE electrical engineering symposium on recent advances (RAEE). Islamabad, Pakistan, 19-20 Oct 2015.

[10] Chantachiratham P, Hongesombut K (2012) PSO based approach for optimum fault current limiter placement in power system. In: Proceedings of the $20129^{\text {th }}$ international conference on electrical engineering, computer, telecommunications and information technology (ECTI-CON). Phetchaburi, Thailand, 16-18 May 2012, pp 1-4.

[11] Zare S, Khazali A, Hashemi SM et al (2013) Fault current limiter optimal placement by harmony search algorithm. In: Proceedings of the $201322^{\text {nd }}$ international conference and exhibition on electricity distribution (CIRED 2013). Stockholm, Sweden, 10-13 June 2013.

[12] Najy WKA, Zeineldin HH, Woon WL (2013) Optimal protection coordination for microgrids with grid-connected and islanded capability. IEEE Trans Ind Electron 60(4):1668-1677

[13] Zhao Y, Krause O, Saha TK (2015) A new approach for optimal allocation of multiple SFCLs in a power system with distributed generation. In: Proceedings of the 2015 IEEE power and energy engineering conference (APPEEC). Brisbane, Australia, 15-18 Nov 2015

[14] Nie S, Tian L, Qi D (2014) Optimal allocation of superconducting fault current limiters considering voltage sags mitigation. In: Proceedings of the 2014 IEEE PES Asia-Pacific power and energy engineering conference (APPEEC). Hong Kong, China, 7-10 Dec 2014

[15] Alaraifi S, El Moursi MS, Zeineldin HH (2013) Optimal allocation of HTS-FCL for power system security and stability enhancement. IEEE Trans Power Syst 28(4):4701-4711

[16] Yu P, Venkatesh B, Yazdani A et al (2016) Optimal location and sizing of fault current limiters in mesh networks using iterative mixed integer nonlinear programming. IEEE Trans Power Syst. doi:10.1109/TPWRS.2015.2507067

[17] Huchel L, Zeineldin H, El-Saadany E (2016) Protection coordination index enhancement considering multiple DG locations using FCL. IEEE Trans Power Deliv. doi:10.1109/TPWRD. 2016.2533565

[18] Teng JH, Lu CN (2010) Optimum fault current limiter placement with search space reduction technique. IET Gener Transm Distrib 4(4):485-494

[19] Rui MA, Kai LI, Xuan LI (2015) An economic and low-carbon day-ahead Pareto-optimal scheduling for wind farm integrated power systems with demand response. J Mod Power Syst Clean Energy 3(3):393-401. doi:10.1007/s40565-014-0094-7

[20] Khaleghi M, Farsangi MM, Nezamabadi-pour H et al (2011) Pareto-optimal design of damping controllers using modified artificial immune algorithm. IEEE Trans Syst Man Cybern Part C 41(2):240-250
[21] Yuan JX, Pan JB, Fei WL (2013) An immune-algorithm-based space-vector PWM control strategy in a three-phase inverter. IEEE Trans Ind Electron 60(5):2084-2093

[22] Chang GW, Chang WC, Chuang CS et al (2011) Fuzzy logic and immune-based algorithm for placement and sizing of shunt capacitor banks in a distorted power network. IEEE Trans Power Deliv 26(4):2145-2153

Baichao CHEN received the B.E. degree from Huazhong University of Science and Technology, Wuhan, China, in 1982, and the M.S. and Ph.D. degrees from the School of Electrical Engineering, Wuhan University, Wuhan, in 1989 and 1993, respectively. Currently, he is a Professor of Wuhan University, focusing on the magnetic valve controlled reactor, the saturated-core fault current limiter and the reactive power compensation technology.

Liangliang WEI received the B.S. and M.S. degrees in Electrical Engineering from Wuhan University Wuhan China in 2012 and 2014 respectively. Currently he is pursuing the $\mathrm{Ph} . \mathrm{D}$. degree in electrical engineering with an emphasis on the fault current limiter. His research interests include the fault current limiter and the reactive power compensation technology.

Yuanzhe ZHU received the B.S. degrees in Electrical Engineering from Wuhan University, Wuhan, China, in 2016, where he is currently pursuing M.S. degree in Electrical Engineering. His research interests include the fault current limiter and power filter.

Yongheng ZHONG received the B.S. degrees in Electrical Engineering from Wuhan University, Wuhan, China, in 2015, where he is currently pursuing M.S. degree in Electrical Engineering. His research interests include the fault current limiter based on permanent magnets and power quality of railway power supply system.

Jiaxin YUAN received the B.S. and Ph.D. degrees in Electrical Engineering from the Wuhan University, Wuhan, China, in 2002 and 2007, respectively. From 2007 to 2009, he was a lecturer in Wuhan University, where he has been engaged in power electronics system control, power quality issues, application and control of inverters. From 2014 to 2015, he was a visiting scholar at the Electrical and Computer Engineering Ohio state university, Columbus, OH. Since 2009, He is an Associate Professor in electrical engineering from Wuhan University. Dr. Yuan is an active member of IEEE. He was the author of more than 70 scientific publications, among which, 18 papers were published or accepted in IEEE transactions or IET proceedings and held 33 patents. His research interests include the wide band gap device based power electronics circuits and control, interface for renewable energy sources, and Flexible AC Transmission System devices.

Yang LEI received the B.S. and M.S. degrees in Electrical Engineering from Wuhan University, Wuhan, China, in 2012 and 2016, respectively. His main research interests include the fault current limiter and distributed generation. 